\title{
Interesses Profissionais Expressos e Inventariados de Estudantes de Psicologia: Implicações para a Formação*
}

\section{Expressed and Inventoried Vocational Interest of Psychology Students: Implications for Training}

\author{
Intereses Profesionales Expressados e Inventariados de los Estudiantes de Psicología: \\ Implicaciones para la Formación
}

\author{
Rodolfo Augusto Matteo Ambiel \\ Universidade São Francisco. e-mail: ambielram@gmail.com \\ Gustavo Henrique Martins \\ Universidade São Francisco. e-mail: gustavoh.martins95@gmail.com \\ *Agência de fomento: CNPq
}

\section{Resumo}

O objetivo deste estudo foi verificar a relação entre interesses expressos e inventariados de estudantes de Psicologia por áreas de atuação da profissão. Participaram 392 estudantes, predominantemente do sexo feminino e provenientes de instituições particulares do estado de São Paulo. Para a coleta de dados foi utilizada a Escala de Interesses por Áreas da Psicologia (EIAPsi) e um questionário de identificação. Os resultados da análise de variância mostraram que para todos os fatores do instrumento analisados houve discriminação significativa dos grupos de interesses expressos, com tamanhos de efeito das diferenças superiores a 1,00, de forma coincidente com as áreas de preferência marcadas pelos alunos. Os resultados são discutidos considerando as implicações dos resultados para a formação em Psicologia e para a transição para a carreira de psicólogo. Novas pesquisas são sugeridas.

Palavras-chave: avaliação psicológica, ensino superior, orientação vocacional, psicometria.

\section{Abstract}

The aim of this study was to investigate the relationship between expressed and inventoried vocational interests of Psychology students by areas of the profession acting. 392 students participated, predominantly women and from private institutions in the state of São Paulo. For data collection was used Interests Scale for Areas of Psychology (EIAPsi) and an identification questionnaire. The results of analysis of variance showed that for all instrument factors analyzed significant discrimination against groups expressed interest, with effect sizes of differences of more than 1.00 , coinciding with the preferred areas marked by students. The results are discussed considering the implications of the results for training in Psychology and the transition to the psychologist's career. Further studies are suggested.

Keywords: psychological assessment, higher education, vocational counseling, psychometrics.

Resumen

El objetivo de este estudio fue investigar la relación entre los intereses profesionales de estudiantes de Psicología expresada e inventariada por áreas da Psicología. 392 estudiantes participaron, mayormente mujeres y de las instituciones privadas en el estado de São Paulo. Para la recolección de datos se utilizó intereses Escala de Espacios de Psicología (EIAPsi) y un cuestionario de identificación. Los resultados del análisis de varianza mostraron que todos los factores analizados instrumento importante discriminación contra los grupos expresaron su interés, con tamaños del efecto de las diferencias de más de 1,00, coincidiendo con las zonas preferidas por los estudiantes marcados. Los resultados se discuten considerando las implicaciones de los resultados para la formación en la Psicología y la transición a la carrera del psicólogo. Se sugiere además la investigación.

Palabras-clave: evaluación psicológica, educación superior, orientación professional, psicometría. 
Os interesses profissionais têm sido concebidos como padrões de gosto, aversão ou indiferenças em relação aos estímulos acadêmicos ou profissionais (Lent, Brown, \& Hackett, 1994). Recentemente, Rounds e Su (2014) definiram os interesses profissionais como preferências por atividades, como também pelos seus contextos e resultados, os quais motivam comportamentos para alcançar objetivos, mostrando-se assim um poderoso preditor de desempenho e sucesso.

Ainda em relação à conceituação, Silvia (2001) classifica os interesses profissionais em duas concepções, quais sejam os interesses expressos e interesses inventariados ou medidos, definindoos de forma distinta, principalmente a respeito do processo de construção pessoal. Para o autor os interesses expressos são definidos como respostas a perguntas diretas, como por exemplo: O que você vai ser quando crescer? Neste caso, os interesses podem ser incomuns ou inusitados, especialmente na infância ou adolescência, mas que na vida adulta refletem intenções de engajamento em certos campos, a partir de concepções individuais sobre o mundo do trabalho. Por outro lado, os interesses inventariados são representados por respostas a um questionário de interesse global, a partir de uma teoria e interpretadas de forma normativa com base nas respostas de um grupo maior previamente avaliado. Um exemplo de estudo que buscou avaliar a relação entre esses tipos de interesse é o de Noronha, Ambiel, Frigatto, e Toledo (2010), que verificaram haver concordância entre as profissões de intenção de estudantes do ensino fundamental e médio, medida por meio de uma pergunta aberta sobre qual a profissão o estudante escolheria, no momento da coleta, e os escores nos fatores da Escala de Aconselhamento Profissional (EAP).

Nesse sentido, a intenção profissional futura funciona como uma guia que conduz os comportamentos exploratórios presentes e de planejamento, sendo que a obtenção do diploma de um curso superior aparece como o objetivo de vida de muitas pessoas (Lemos, Neves, \& Rodrigues, 2013). Assim sendo, o constante aumento do número de instituições de ensino superior no
Brasil, ultrapassando a marca de 2400 instituições distribuídas entre universidades públicas e privadas (Instituto Nacional de Estudos e Pesquisas Educacionais Anísio Teixeira [INEP], 2014), junto a uma série de programas de incentivo e inclusão oferecidos pelo governo federal (Ministério da Educação [MEC], 2014), favorecem uma maior disponibilidade de vagas no ensino superior à população. Um dado que reforça essa afirmação é que nos últimos 20 anos aumentou em mais de quatro vezes o número de estudantes matriculados no ensino superior brasileiro (Neves, 2012), ainda que isso não implique que a proporção de concluintes seja equivalente (INEP, 2014).

Contudo, ingressar na universidade traz desafios afetivos, cognitivos e sociais. Os anos que os estudantes passam na universidade são importantes tanto para o desenvolvimento pessoal quanto para a formação profissional, refletindo no desenvolvimento da própria sociedade onde irão atuar quando graduados (Lamas, Ambiel, \& Silva, 2014; Magalhães, 2013; Sarriera, Paradiso, Schütz, \& Howes, 2012).

Durante a vivência como estudante universitário, até a inserção no mercado de trabalho, pode haver um período caracterizado como exploratório (Super, Savickas, \& Super, 1996), que tem por finalidade experimentar diferentes papéis ligados às possibilidades existentes em sua futura profissão. Sendo assim, alguns cursos exigem do estudante a realização de escolhas por áreas de estágio ou atuação ainda durante o curso, a partir de diversas áreas disponíveis dentro de uma mesma profissão (Magalhães \& Teixeira, 2013; Silva, Coelho \& Teixeira, 2013; Teixeira \& Gomes, 2004). Nessa direção, a Psicologia é uma das profissões que faz essa exigência do estudante, por apresentar uma grande diversidade de opções de áreas de atuação (Bedin, Sarriera, \& Paradiso, 2013).

Esse quadro sugere que os estudantes de Psicologia deveriam ter contato com toda a diversidade de campos de atuação para que se tenha uma melhor tomada de decisão em relação à carreira profissional, uma vez que o estudante se depara com algumas situações durante o 
curso que lhe geram incertezas quanto à escolha, necessitando assim de algum mecanismo de auxilio para a superação deste conflito (Campos, Catão, \& Fuji, 1999). Segundo dados relatados por Achcar (1994), dentre os aspectos que impactam na formação do psicólogo, a identidade social parece ter forte influência, apontando que muitos estudantes acreditam que somente serão plenamente psicólogos aqueles que atuarem na área Clínica. Essa percepção é corroborada por Bosi e Elias (2000), Magalhães, Straliotto, Keller, e Gomes (2001) e Bueno, Lemos, e Tomé (2004), cujos estudos apontam que os motivos que levam os estudantes a optar pelo curso de Psicologia parecem relacionados à busca de uma melhor condição ou de um novo caminho na vida, à busca de crescimento pessoal e/ou competência interpessoal, ao fascínio pelo conhecimento psicológico e ao desejo de compreender e ajudar o ser humano. Nesse sentido, mesmo em outros países, as áreas mais procuradas no âmbito dos cursos de Psicologia ainda tendem a ser as mais tradicionais, ou seja, Psicologia Clínica, Psicologia das Organizações e Psicologia Educacional (Haselhuhn \& Cloopton, 2008; Lopes, Palma, Bártolo-Ribeiro \& Cunha, 2011; Yamamoto \& Costa, 2010).

Apesar da importância dada aos campos de atuação profissional dos psicólogos, bem como aos estágios de formação durante a graduação, as instituições têm negligenciado o processo de escolha dos estudantes acerca do tema, tanto que não foram encontrados na literatura relatos de experiências sobre programas de ajuda aos estudantes graduandos para escolher suas áreas de estágio, tampouco de planejamento de seu futuro profissional. Possivelmente isso seja estimulado pelas próprias Diretrizes Curriculares Nacionais (DCN), publicadas pelo MEC (2011), que estimulam a formação generalista do profissional em nível de graduação, isto é, que o psicólogo seja formado para a atuação em qualquer área possível. Já a respeito da formação pós-graduada, embora a literatura científica também seja omissa a esse respeito, o Conselho Federal de Psicologia (2007), por meio da Resolução CFP 013/2007, reconheceu e sistematizou 11 campos de especialização, quais sejam, Escolar/Educacional, Organizacional/ Trabalho, Trânsito, Jurídica, Esporte, Clínica, Hospitalar, Psicopedagogia, Psicomotricidade, Social e Neuropsicologia. Vale ressaltar, contudo, que no Brasil as especializações são reguladas pelo $\mathrm{MEC}$, que possibilita especializações em áreas que o CFP não reconhece, tal como a avaliação psicológica (Noronha \& Reppold, 2010).

Assim, uma das contribuições possíveis para essa situação de escolha por áreas de atuação, é o desenvolvimento de instrumentos que possam auxiliar no processo de escolha, por exemplo, promovendo autoconhecimento acerca das motivações e padrões de preferência por atividades profissionais do psicólogo, ou seja, de seus interesses profissionais. Alguns autores, preocupados com as dificuldades vocacionais e de carreira aparentes nos estudantes, se propuseram a investigar os aspectos de interesses profissionais por parte dos estudantes de Psicologia.

Por exemplo, Campos et al. (1999), adaptaram para o português brasileiro um instrumento americano denominado Scientist practitioner Inventory (Inventário cientista-prático). A pesquisa teve por objetivo identificar a tendência de um grupo de alunos de Psicologia quanto à área de atuação e o instrumento adaptado foi aplicado em um grupo de 46 estudantes de Psicologia de uma universidade particular de São Paulo. $O$ instrumento dispunha de 42 itens, sendo 13 relativos a atividades terapêuticas, 10 à pesquisa, 05 ao perito em clínica, 04 à estatística, 04 a professor - editor - supervisor, 03 a ideias acadêmicas e 03 a testes e interpretação. Com os resultados obtidos, os autores concluíram que o maior interesse por parte destes estudantes está contido na área clínica, sendo o contrário observado quanto à área da estatística. Os autores concluíram também que este instrumento foi baseado no modelo norte-americano cientistaprático, desconsiderando as demais áreas e sugerindo assim novos estudos com base na realidade brasileira.

Em Portugal, Rodrigues, Ferreira, e BártoloRibeiro (2013) desenvolveram um questionário de interesses pela Psicologia com 21 itens, decorrentes de um levantamento teórico. Para a construção 
do instrumento foi utilizada uma amostra de 573 estudantes de Psicologia e, por meio da análise de componentes principais, extraíram três dimensões, Organizacional, Educacional e Clínica. Mesmo com bons índices de consistência interna, variando de 0,86 a 0,93, os autores sugerem o desenvolvimento de novos estudos, a fim de validar e confirmar os resultados obtidos. Em outro estudo português, Ferreira, Rodrigues, e Ferreira (2015) encontraram correlações positivas e significativas entre as dimensões Clínica e Educacional e o tipo Social do modelo RIASEC e entre a dimensão Organizacional com o tipo Empreendedor.

Como abordado até aqui, nota-se que os instrumentos apresentados, mostraram-se restritos em suas avaliações em relação à totalidade de áreas existentes na Psicologia, corroborando a necessidade de novos instrumentos que abordem de maneira mais ampla este espectro, em que pese as diferenças culturais e de formação em diferentes países. Partindo deste princípio, Ambiel e Zanon (2013) construíram a Escala de Interesses por Áreas da Psicologia (EIAPsi) em uma disciplina de graduação em Psicologia de uma universidade particular no interior paulista, no qual os estudantes deveriam criar itens de interesses por áreas da Psicologia, baseados inicialmente na Resolução CFP No 013/2007, por se considerar que, independente de implicações legais do documento, as informações ali contidas eram bastante detalhadas e completas para a tarefa.

Após a construção da escala, foi realizada uma avaliação da validade de conteúdo com oito psicólogos de diferentes áreas de atuação e de ambos os sexos, a qual indicou índices satisfatórios de concordância a respeito do conteúdo da EIAPsi (Martins, Hernández, \& Ambiel, 2014). Em seguida foi realizado um estudo piloto, que contou com 296 participantes, dentre eles 170 estudantes de Psicologia e 126 psicólogos formados. Foi realizada uma Análise Fatorial Exploratória, que permitiu apurar o agrupamento de 61 dos 91 itens iniciais em oito fatores, quais sejam Organizacional, Educacional, Esporte, Avaliação Psicológica,
Saúde, Jurídica, Neuropsicologia e Trânsito, com precisão variando entre 0,88 (Neuropsicologia) a 0,96 (Organizacional) (Martins \& Ambiel, 2015).

Apesar dos bons resultados iniciais, três fatores esperados não foram observados, relacionados à Psicologia Clínica, Social e, Docência e Pesquisa (Martins \& Ambiel, 2015). Portanto, a partir dos resultados iniciais, percebeu-se a necessidade da formulação de novos itens para cobrir as áreas que não foram representadas. Com isso, foi aplicada uma nova versão da escala com 90 itens, a qual contou com a participação de 392 estudantes de Psicologia, que por meio da Análise Fatorial Exploratória, apontou para a existência de 11 fatores para a EIAPsi, sem a necessidade da exclusão de nenhum dos itens da escala. Além disso, foi constatada a boa consistência interna dos fatores, que variou de 0,84 (Clínica e Avaliação Psicológica) a 0,95 (Organizacional). Portanto, o instrumento mostrou-se apto para avaliar os interesses de estudantes de Psicologia pelas áreas Organizacional, Docência e Pesquisa, Esporte, Jurídica, Social, Educacional, Saúde, Avaliação Psicológica, Clínica, Neuropsicologia e Trânsito. Entretanto, mesmo com as boas qualidades psicométricas constatadas, novas evidências de validade da EIAPsi ainda são necessárias (Ambiel \& Martins, 2016).

Nesta direção, pretende-se com este estudo realizar uma comparação entre os interesses expressos e os interesses inventariados dos estudantes a respeito das áreas de atuação da profissão. Como apontado em estudos anteriores, os interesses expressos medidos por meio de perguntas diretas a respeito das intenções profissionais estão comumente relacionados com os fatores de interesses equivalentes medidos por meio de inventários (interesses inventariados) (Noronha et al., 2010; Silvia, 2001). Sendo assim, propõe-se a seguinte hipótese:

H1. Os interesses inventariados pela EIAPsi (i.e., pontuações nos fatores) discriminarão os grupos formados a partir dos interesses expressos (i.e., campos de atuação), com tamanhos de efeitos grandes. 


\section{Método}

\section{Participantes}

A amostra deste estudo é composta por 392 estudantes de Psicologia de diversas regiões do Brasil, com destaque para os Estados de São Paulo (71,4\%), Santa Catarina (11,5\%), Bahia (10,2\%) e Minas Gerais (4,3\%) e predominantemente de instituições particulares $(94,9 \%)$. A maior parte dos estudantes é do sexo feminino (77,6\%). A idade dos participantes variou de 18 a 60 anos, com média de idade de 24,19 ( $D P=7,02)$. Em relação ao semestre foi observado a seguinte distribuição, $1^{\circ}$ (0,3\%), $2^{\circ}$ (3,3\%), $3^{\circ}(7,7 \%), 4^{\circ}$ (25\%), $5^{\circ}(13 \%), 6^{\circ}(22,2 \%), 7^{\circ}(3,1 \%), 8^{\circ}(3,8 \%)$, $9^{\circ}(7,1 \%)$ e $10^{\circ}(9,7 \%)$ semestres. A coleta de dados ocorreu de forma presencial no formato lápis e papel ( $n=235)$ e online por meio do aplicativo Google Drive ( $n=157)$, sendo que não há efeito importante do tipo de aplicação sobre as respostas dos participantes $(d=0,18)$.

\section{Instrumentos}

Questionário de identificação: composto exclusivamente para este projeto; é formado por itens que buscaram avaliar as características sociodemográficas e acadêmicas dos participantes. Além disso, verificou a área de intenção dos estudantes, os quais tiveram que escolher, em uma lista, qual a área de maior preferência para atuar quando formados, a fim de responder a seguinte pergunta: "Em relação à área que pretende atuar quando formado, anote aquela que você tem mais interesse". Sendo assim, as opções foram: Jurídica, Psicopedagogia, Neuropsicologia, Organizacional/ Trabalho, Docência e/ou Pesquisa, Social, Saúde, Clínica, Trânsito, Esporte, Avaliação Psicológica, Psicomotricidade, Escolar/Educacional, "Outra”.

Escala de Interesses por Áreas da Psicologia - EIAPsi (Ambiel \& Martins, 2016): esta escala avalia os interesses de estudantes ou profissionais da Psicologia com as áreas possíveis de atuação do Psicólogo. O examinado deve responder aos itens em uma escala do tipo Likert de 5 pontos, com variações entre 'detesto/detestaria' (1) e 'adoro/ adoraria' (5). A escala é composta por 90 itens, representados em 11 fatores: Organizacional (11 itens - ex. Responsabilizar-se em melhorar as condições de trabalho na organização- $\alpha=0,95$ ), Docência e Pesquisa (12 itens - ex. Orientar trabalhos de alunos de iniciação científica, mestrado e doutorado $-\alpha=0,91$ ), Esporte (oito itens - ex. Realizar atendimentos com o intuito de preparar o atleta para o desempenho da atividade $-\alpha=0,93$ ), Jurídica (oito itens - ex. Elaborar laudos sobre o funcionamento mental dos indivíduos em processos judiciais $-\alpha=0,89$ ), Social (nove itens - ex. Atuar na atenção a grupos em situação de vulnerabilidade em centros comunitários $\alpha=0,89$ ), Educacional (nove itens - ex. Analisar e intervir no clima educacional $-\alpha=0,89$ ), Saúde (oito itens - ex. Atuar junto a pacientes terminais hospitalizados $-\alpha=0,91$ ), Avaliação Psicológica (sete itens - ex. Possibilitar a tomada de decisão a partir de avaliações $-\alpha=0,84$ ), Clínica (sete itens - ex. Realizar atendimentos psicoterapêuticos em consultórios ou clínicas particulares $-\alpha=0,84$ ), Neuropsicologia (seis itens - ex. Realizar avaliações das funções neuropsicológicas $-\alpha=0,88$ ) e Trânsito (cinco itens - ex. Avaliar capacidades, habilidades e aptidões de candidatos à carteira de motorista $-\alpha=0,90)$.

\section{Procedimentos}

O projeto foi submetido e aprovado pelo Comitê de Ética em Pesquisa (CEP) da Universidade São Francisco (número do Parecer: 770.173). Primeiramente, deu-se início a coleta presencial e, posteriormente, foi adotada a estratégia online a fim de se ampliar a diversidade da amostra em diferentes estados. A coleta presencial ocorreu em sala de aula de forma coletiva, em formato lápis e papel, e a coleta online ocorreu por meio do aplicativo Google Drive a partir do encaminhamento do e-mail contendo o convite da pesquisa aos docentes responsáveis, que 
disponibilizaram aos estudantes de Psicologia o link da pesquisa junto com o convite para sua participação. A participação na pesquisa só foi permitida a partir da concordância do participante com o Termo de Consentimento Livre e Esclarecido (TCLE).

\section{Análise de dados}

Após a coleta, os dados foram tabulados em planilhas do SPSS. Inicialmente, foram feitas análises descritivas dos participantes, em termos das variáveis sociodemográficas e acadêmicas, a fim de descrever a amostra. Em seguida foi realizada uma Análise de Variância (ANOVA) com provas post-hoc de Tukey $(\mathrm{p} \leq 0,01)$, entre os fatores da EIAPsi e as áreas de intenção dos estudantes. Posteriormente, foram criadas variáveis dicotômicas para cada área de intenção avaliada, indicando se o participante apresentou ou não intenção por uma área específica. Por exemplo, o grupo que expressou interesses pela área organizacional recebeu o label 1 , enquanto todos os outros participantes receberam 0 . Dessa forma, foram criados dois grupos para cada área listada. A partir dessa categorização, foi verificado o tamanho do efeito (effect-size) das diferenças entre os grupos, sendo interpretados de acordo com Cohen (1988), ou seja, tamanhos de efeito pequeno $d=0,2$, médio $d=0,5$ e grande $d=0,8$.

\section{Resultados}

Nessa sessão, serão apresentadas as estatísticas descritivas acerca dos interesses expressos dos participantes e posteriormente as comparações entre os grupos. A Tabela 1 refere-se às frequências das áreas de intenção assinaladas pelos estudantes de Psicologia.
Foi adotado, como critério arbitrário para permanência das áreas de intenção, o número de pelo menos 20 respostas em cada opção, para assegurar um mínimo de variabilidade nas respostas. Quando esse total não foi atingido, buscou-se agrupar áreas conforme a semelhança

Tabela 1

Frequência das Áreas de Intenção.

\begin{tabular}{lcc}
\hline Opção & Frequência & $\%$ \\
\hline Psicomotricidade & 2 & 0,5 \\
Esporte & 5 & 1,3 \\
Avaliação Psicológica & 6 & 1,5 \\
Trânsito & 10 & 2,6 \\
Psicopedagogia & 12 & 3,1 \\
Escolar/Educacional & 16 & 4,1 \\
Neuropsicologia & 23 & 5,9 \\
Social & 26 & 6,6 \\
Docência e/ou Pesquisa & 31 & 7,9 \\
Saúde & 51 & 13,0 \\
Jurídica & 53 & 13,5 \\
Organizacional/Trabalho & 61 & 15,6 \\
Clínica & 76 & 19,4 \\
Outra & 1 & 0,3 \\
Ausente & 19 & 4,8 \\
Total & 392 & 100 \\
\hline
\end{tabular}


de atividades ou, quando isso não era possível, o campo foi excluído. A partir disso, foram agrupadas as respostas das áreas Educacional e Psicopedagogia (16 e 12 respostas, respectivamente). Em seguida, foram excluídas as opções pelas áreas Trânsito, Esporte, Avaliação Psicológica, Psicomotricidade e a opção "Outra", pois nenhuma destas opções apresentou quantidade suficiente de respostas.
Portanto as áreas de intenção consideradas nas análises foram Jurídica, Escolar/Psicopedagogia, Neuropsicologia, Organizacional, Docência e Pesquisa, Social, Saúde e Clínica. Os resultados serão divididos em duas tabelas para melhor compreensão. A seguir, na Tabela 2, serão apresentados os dados da ANOVA e da prova de Tukey dos fatores Organizacional, Docência e

\section{Tabela 2}

Análise de variância e prova de Tukey para os fatores da EIAPsi e as áreas de intenção.

\begin{tabular}{|c|c|c|c|c|c|c|}
\hline \multirow[b]{2}{*}{ Fatores/Áreas da EIAPsi } & \multirow[b]{2}{*}{$F^{*}$} & \multirow[b]{2}{*}{ Interesses expressos } & \multicolumn{4}{|c|}{ Subconjuntos** } \\
\hline & & & 1 & 2 & 3 & 4 \\
\hline \multirow[t]{8}{*}{ Organizacional } & 25,612 & Neuropsicologia & 29,52 & & & \\
\hline & & Saúde & 29,69 & 29,69 & & \\
\hline & & Social & 30,46 & 30,46 & & \\
\hline & & Docência e Pesquisa & 31,32 & 31,32 & & \\
\hline & & Jurídica & 32,00 & 32,00 & & \\
\hline & & Clínica & 32,39 & 32,39 & & \\
\hline & & Escolar & & 36,61 & & \\
\hline & & Organizacional & & & 49,52 & \\
\hline \multirow[t]{8}{*}{ Docência e Pesquisa } & 10,322 & Escolar & 34,75 & & & \\
\hline & & Jurídica & 36,19 & & & \\
\hline & & Clínica & 36,97 & & & \\
\hline & & Saúde & 37,71 & & & \\
\hline & & Organizacional & 39,25 & & & \\
\hline & & Neuropsicologia & 40,43 & & & \\
\hline & & Social & 41,23 & & & \\
\hline & & Docência e Pesquisa & & 52,29 & & \\
\hline \multirow[t]{8}{*}{ Jurídica } & 16,723 & Escolar & 21,64 & & & \\
\hline & & Neuropsicologia & 23,35 & 23,35 & & \\
\hline & & Docência e Pesquisa & 23,39 & 23,39 & & \\
\hline & & Clínica & 23,68 & 23,68 & & \\
\hline & & Organizacional & 23,95 & 23,95 & & \\
\hline & & Saúde & 25,37 & 25,37 & & \\
\hline & & Social & & 26,69 & & \\
\hline & & Jurídica & & & 34,13 & \\
\hline \multirow[t]{8}{*}{ Neuropsicologia } & 5,989 & Docência e Pesquisa & 19,13 & & & \\
\hline & & Organizacional & 19,38 & & & \\
\hline & & Escolar & 19,71 & & & \\
\hline & & Social & 19,73 & & & \\
\hline & & Jurídica & 20,04 & & & \\
\hline & & Clínica & 20,36 & & & \\
\hline & & Saúde & 20,75 & & & \\
\hline & & Neuropsicologia & & 27,04 & & \\
\hline
\end{tabular}

${ }^{*} g l=7 ;{ }^{* *} p<0,01$ 
Pesquisa, Jurídica e Neuropsicologia.

Foi constatado que, nos quatro fatores analisados, a área de intenção equivalente ao fator apresentou diferenciação significativa das demais áreas de intenção, formando um subconjunto isolado que se diferenciou dos outros subconjuntos. É importante também destacar que no fator Organizacional a área de intenção que menos pontuou foi a Neuropsicologia, no fator
Docência e Pesquisa a área que menos pontuou foi a Escolar/Psicopedagogia, no fator Jurídica, a área Escolar/Psicopedagogia e por fim, no fator Neuropsicologia, a área que menos pontuou no fator foi a Docência e Pesquisa. A seguir, na Tabela 3 , serão apresentados os dados da ANOVA e da prova de Tukey dos fatores Educacional, Saúde, Clínica e Social.

Novamente, nos quatro fatores analisados, as

\section{Tabela 3}

Análise de variância e prova de Tukey para os fatores da EIAPsi e as áreas de intenção.

\begin{tabular}{|c|c|c|c|c|c|c|}
\hline \multirow[b]{2}{*}{ Fatores/Áreas da EIAPsi } & \multirow[b]{2}{*}{$\boldsymbol{F}^{*}$} & \multirow[b]{2}{*}{ Interesses expressos } & \multicolumn{4}{|c|}{ Subconjuntos** } \\
\hline & & & 1 & 2 & 3 & 4 \\
\hline \multirow[t]{8}{*}{ Educacional } & 9,300 & Neuropsicologia & 27,39 & & & \\
\hline & & Docência e Pesquisa & 28,13 & & & \\
\hline & & Jurídica & 28,19 & & & \\
\hline & & Clínica & 28,97 & & & \\
\hline & & Organizacional & 29,07 & & & \\
\hline & & Saúde & 30,06 & & & \\
\hline & & Social & 32,04 & & & \\
\hline & & Escolar & & 40,89 & & \\
\hline \multirow[t]{8}{*}{ Saúde } & 15,939 & Docência e Pesquisa & 21,81 & & & \\
\hline & & Organizacional & 24,54 & 24,54 & & \\
\hline & & Neuropsicologia & 25,96 & 25,96 & 25,96 & \\
\hline & & Escolar & & 27,46 & 27,46 & \\
\hline & & Jurídica & & 27,68 & 27,68 & \\
\hline & & Clínica & & 28,92 & 28,92 & \\
\hline & & Social & & & 30,35 & \\
\hline & & Saúde & & & & 35,53 \\
\hline \multirow[t]{8}{*}{ Clínica } & 9,822 & Docência e Pesquisa & 23,94 & & & \\
\hline & & Jurídica & 24,83 & 24,83 & & \\
\hline & & Organizacional & 25,44 & 25,44 & & \\
\hline & & Social & 25,85 & 25,85 & & \\
\hline & & Neuropsicologia & 27,00 & 27,00 & 27,00 & \\
\hline & & Escolar & 27,11 & 27,11 & 27,11 & \\
\hline & & Saúde & & 27,78 & 27,78 & \\
\hline & & Clínica & & & 30,50 & \\
\hline \multirow[t]{8}{*}{ Social } & 8,810 & Neuropsicologia & 25,43 & & & \\
\hline & & Docência e Pesquisa & 28,68 & 28,68 & & \\
\hline & & Clínica & 29,51 & 29,51 & & \\
\hline & & Saúde & 29,61 & 29,61 & & \\
\hline & & Jurídica & 30,02 & 30,02 & & \\
\hline & & Organizacional & & 30,59 & & \\
\hline & & Escolar & & 33,50 & & \\
\hline & & Social & & & 39,50 & \\
\hline
\end{tabular}

${ }^{*} g l=7 ;{ }^{* *} p<0,01$ 
áreas que tiveram as maiores pontuações foram as áreas de intenção equivalentes ao fator. Pode-se destacar que embora a área Clínica tenha sido a única das outras áreas de intenção que não formou um subconjunto isolado, foi possível observar que esta área se diferenciou de todos os demais subconjuntos. Entretanto, destaca-se que no fator Educacional a menor pontuação foi da área de intenção Neuropsicologia, no fator Saúde a menor pontuação foi da área Docência e Pesquisa, no fator Clínica a menor pontuação foi da área Docência e Pesquisa e por fim, no fator Social a área de intenção que apresentou menor pontuação foi a Neuropsicologia.

Em suma, os resultados apresentados, na Tabela 2 e na Tabela 3 , indicaram que em todos os casos analisados as diferenças entre os grupos foram significativas $(p<0,01)$, sendo que os participantes que marcaram a área de intenção equivalente ao fator da EIAPsi obtiveram as maiores médias no respectivo fator, diferenciando-se claramente dos outros subconjuntos das demais áreas. Em outras palavras, os estudantes de Psicologia que informaram ter maior intenção de atuar na área
Organizacional, por exemplo, apresentaram maiores pontuações no fator Organizacional da EIAPsi, dado que esse fato foi igualmente constatado em todas as outras áreas analisadas.

Em seguida, buscou-se refinar esses resultados por meio da análise do tamanho das diferenças entre os grupos das áreas de intenção nos equivalentes fatores da EIAPsi. Para tanto, houve o agrupamento dicotômico de interesse ou não-interesse pelas áreas. Assim, foi possível constatar os seguintes coeficientes $d$ de Cohen: Organizacional $d=2,07$, Docência e Pesquisa $d=1,67$, Jurídica $d=1,65$, Neuropsicologia $d=1,58$, Educacional $d=1,79$, Saúde $d=1,41$, Clínica $d=1,04$ e Social $d=1,66$. Todos os valores encontrados denotam tamanhos de efeito grandes, indicando que os grupos de participantes que marcaram ter intenção pelas áreas equivalentes aos fatores da EIAPsi apresentaram um efeito muito maior nas médias das pontuações totais do fator da EIAPsi em comparação com os grupos de participantes que não marcaram ter intenção de atuar nestas áreas.

\section{Discussão}

O objetivo do presente estudo foi testar a hipótese de que os interesses profissionais de estudantes de Psicologia, tais como avaliados pela EIAPsi, discriminariam os grupos formados a partir dos interesses expressos por meio de uma lista de possíveis campos de atuação da Psicologia. Os resultados suportaram amplamente tal hipótese, de forma que o objetivo da pesquisa foi atingido. Importante salientar que os resultados foram favoráveis, tanto quando foram comparados todos os grupos de interesse de forma independente (ANOVA), quanto quando os grupos foram dicotomizados e contrastados ( $d$ de Cohen). Os achados serão discutidos a seguir.

Foi constatado que em todos os fatores da EIAPsi foram observadas diferenças significativas entre os grupos das áreas de intenção dos estudantes. Por meio da prova de Tukey, foi verificado que em sete dos oito fatores comparados as áreas de intenção equivalentes formaram um subconjunto isolado em relação as demais áreas. Reforçando tal afirmação, os dados provenientes dos tamanhos de efeitos das diferenças entre os grupos dicotomizados também esclarece que a EIAPsi mostrou-se válida para identificar as áreas de maior interesse dos estudantes. Importante notar que esses achados gerais vão ao encontro de resultados anteriores, tais como os de Noronha et al. (2010), Rounds e Su (2014), e Silvia (2001) ao constatar as relações entre os interesses expressos com os inventariados.

A área Clínica foi a única que não formou um subconjunto isolado, compondo-o com mais três áreas. Destas, tanto a Neuropsicologia quanto a Escolar/Psicopedagogia não se diferenciaram de nenhum dos três subconjuntos formados e a 
área da Saúde se diferenciou apenas do primeiro subconjunto, permanecendo em outros dois. Portanto, as pessoas que assinalaram ter intenção pela área da Saúde, também apresentaram forte interesse na área Clínica pela EIAPsi, fato que pode ser explicado pela semelhança de atividades em ambas as áreas, voltadas à melhoria e restabelecimento da saúde dos indivíduos. Os achados anteriores de Ferreira et al. (2015) e Rodrigues et al. (2013), em que pesam as diferenças entre os instrumentos e aspectos culturais e formativos, já davam indicativos de que esse resultados seria esperado, uma vez que encontraram um grande fator relacionado às atividades clínicas gerais, além de identificarem que altas pontuações nesse fator relacionam-se com a necessidade de cuidar de outras pessoas em situações clínicas.

Outro resultado que vale ser ressaltado é que, para além das áreas de maior preferência, o estudo também permitiu aferir em alguns casos áreas de menor interesse. Nesse sentido, destaca-se que pessoas com menor pontuação no fator Organizacional da EIAPsi foram as que expressaram interesses por Neuropsicologia. Já no fator Jurídica, os que expressaram maior interesse pela área Escolar foram os que menos pontuaram e, reforçando o achado anterior, o grupo com interesse expresso por Docência e Pesquisa pontuaram menos tanto nos fatores Saúde quanto Clínica. Tais resultados, que encontram suporte nos achados de Campos et al. (1999), reforçam a grande diversidade de interesses presentes nos estudantes de Psicologia, como já notado por Bedin et al. (2013), o que é um reflexo da formação generalista da Psicologia brasileira.

Por fim, embora não fosse objetivo do estudo buscar evidências de validade para a EIAPsi, os resultados encontrados acenam também nesse sentido. A possibilidade de discriminar os interesses expressos dos estudantes a partir das pontuações do instrumento sinalizam um bom potencial preditivo, que deverá ser novamente testado em estudos futuros. Nesse contexto, um instrumento com capacidade preditiva pode ser útil ao informar, com certa segurança, que as percepções do estudante encontram respaldo na avaliação, podendo ser usado em processo de autoconhecimento.

Embora os resultados tenham sido favoráveis, é necessário apontar que ao menos três limitaçoes do estudo devem ser ressaltadas. A primeira limitação é relativa à amostra, uma vez que houve uma maior concentração de estudantes de universidades particulares do estado de São Paulo. Assim, para além do fato de que os resultados não podem ser generalizados por questões geográficas, ainda pode-se considerar que pode ter ocorrido um viés nos resultados por conta da cultura formativa de um tipo de dependência adminstrativa em detrimento de outro, se é que existem tais diferenças, uma vez que não há dados na literatura que permitam avançar nessa discussão.

Além disso, é sabido que a região Sudeste e, de modo particular, o estado de São Paulo tem sido historicamente beneficiado com um grande número de professores doutores, concentrando cerca de $50 \%$ dos programas de pós-graduação, independente da modalidade (Borges-Andrade, Bastos, Andery, Guzzo, \& Trindade, 2014). Dessa forma, é possível que haja um impacto na formação dos alunos que os leve a reconhecer melhor as atividades próprias ou mais consistentes de cada área, bem como que as atividades práticas, tais como estágios, propiciem uma visão mais apurada da realidade profissional. Deste modo, no futuro, é fundamental considerar nas coletas de dados com a EIAPsi dados referentes à estrutura curricular das instituições de ensino, bem como da formação do corpo docente para que essa hipótese seja verificada.

Em segundo lugar, é importante marcar também que devido à baixa quantidade de respostas de intenção (interesses expressos) pelas áreas de Trânsito, Esporte e Avaliação Psicológica, não foi possível realizar as análises com os respectivos fatores da EIAPsi. Pode-se entender que a baixa preferência espontânea por tais áreas, possivelmente, também esteja relacionada com a formação e atuação profissional. Considerando que os interesses profissionais são padrões de preferência por algumas atividades em detrimento 
de outras e que o processo de desenvolvimento dos interesses leva em conta as experiências passadas da pessoa com as atividades avaliadas (Lent et al., 1994; Rounds \& Su, 2014), muito provavelmente os alunos participantes desta amostra tiveram poucas experiências ao longo dos cursos com essas áreas. No caso específico da Avaliação Psicológica, o fato da mesma não ter reconhecimento como área de especialização, mas ser entendida com atuação transversal, também pode impactar negativamente no interesse dos alunos em trabalhar com isso no futuro. Além disso, os problemas históricos de formação em Avaliação Psicológica no Brasil já foram amplamente mapeados (Noronha \& Reppold, 2010).

Por fim, o estudo apresentou como limitação a metodologia utilizada para coletar a informação a respeito da área de intenção dos estudantes. Segundo Silvia (2001) os interesses expressos são respostas diretas a perguntas sobre as suas intenções profissionais. Nesta direção, a metodologia utilizada nesta pesquisa sugeriu um rol de opções de áreas de atuação para o estudante escolher a priori, deixando de ser uma questão necessariamente aberta para ser uma questão direta e com respostas estruturadas. Por outro lado, esta questão permitiu a opção "Outra”, não limitando a resposta dos estudantes àquelas alternativas previamente listadas e que de fato não acrescentou informação à análise. Contudo, não se pode perder de vista o fato de que essa estratégia de coleta de dados tenha influenciado de alguma forma a resposta dos participantes.

\section{Considerações finais}

Este estudo contribui com a literatura científica sobre a formação dos estudantes de Psicologia, uma vez que permite uma série de elocubrações acerca desse importante processo. A primeira contribuição do estudo, mais diretamente derivada de seus resultados, é apresentar um instrumento padronizado amplo o suficiente para captar, se não toda, um parte importante da diversidade da atuação profissional do psicólogo brasileiro. Importa ressaltar que a EIAPsi ainda está em processo de construção, embora já conte com boas evidências de validade.

Outra contribuição do estudo, que apesar de ser indiretamente inferida a partir dos resultados, talvez seja a mais importante. Os resultados conduzem à constatação de que, embora haja a possibilidade de perceber a diversidade de atuações possíveis e uma discriminação aparentemente adequada dos alunos, há áreas que os alunos não percebem como sendo possíveis áreas de trabalho. Por esse motivo sugere-se que as universidades ofereçam contato com a maior diversidade de campos de atuação possível, para que assim o estudante possa escolher suas áreas de atuação de forma mais segura. No futuro, deve-se buscar compreender o motivo disso: será que há uma descompensação em relação aos campos de trabalho na formação? Algumas áreas são mais privilegiadas dos que outras nos currículos de forma sistemática nas instituições? Os docentes de disciplinas teóricas têm conseguido fazer as ligações necessárias entre teoria e prática? Os estágios obrigatórios e optativos têm sido suficientes em termos de carga horária e conteúdo para proporcionar aos alunos experiências de trabalho mais próximas possível da realidade?

Além disso, ao disponibilizar o instrumento finalizado no futuro, espera-se oferecer às coordenações de curso e de serviços-escola, além de outros órgãos do governo como o CFP, subsídio para que possam disponibilizar aos alunos intervenções e programas que os auxiliem a melhor planejar e se preparar para seu futuro. No ponto de vista destes autores, a partir do levantamento bibliográfico, conduzido para a realização do projeto amplo que deu origem a este artigo, a transição da formação para o mercado de trabalho tem sido negligenciada ou, na melhor das hipóteses, não tem sido divulgada pelos agentes formadores. Preparar para a carreira também é formar. 


\section{Referências}

Achcar, R. (Org.). (1994). Psicólogo brasileiro: práticas emergentes e desafios para a formação. São Paulo, SP: Casa do Psicólogo, CFP.

Ambiel, R. A. M., \& Martins, G. H. (2016). Escala de interesses por áreas da psicologia. Relatório técnico não publicado.

Ambiel, R. A. M., \& Zanon, C. (2013). Construção dos itens da Escala de Interesses por Áreas da Psicologia. Relatório técnico não publicado.

Bedin, L. M., Sarriera. J. C., \& Paradiso. A. C. (2013). Desenvolvimento de carreira em psicólogos: tarefas evolutivas de estabelecimento. Revista Brasileira de Orientação Profissional, 14(1), 87-98. Recuperado de http://pepsic.bvsalud.org/pdf/rbop/v14n1/09.pdf

Borges-Andrade, J. E., Bastos, A. V. B., Andery, M. A. P. A., Guzzo, R. S. L., \& Trindade, Z. A. (2014). Psicologia brasileira:uma análise de seus desenvolvimento. Universitas Psychologica, 14(3), 865-880. doi: 10.11144/ Javeriana.upsy14-3.pbua

Bosi, M. L. M., \& Elias, T. F. (2000). Um novo caminho: perfil e trajetórias de alunos de psicologia ingressos como portadores de diploma. Estudos de Psicologia, PUC Campinas, 17(2), 31-40. doi: 10.1590/S0103166X2000000200003

Bueno, J. M. H., Lemos, C. G., \& Tomé, F. A. M. F. (2004). Interesses profissionais de um grupo de estudantes de psicologia e suas relações com inteligência e personalidade. Psicologia em Estudo, 9(2), 271-278. Recuperado de http://www.scielo.br/pdf/pe/v9n2/ v9n2a13

Campos, L. F. L., Catão, E. C., \& Fujii, C. M. (1999). Inventário cientista-prático de orientação profissional em psicologia: um estudo exploratório. Psicologia Escolar e Educacional, 3(2), 139-150. Recuperado de http://pepsic.bvsalud.org/scielo.php?script=sci_ arttext\&pid=S1413-85571999000200004

Cohen, J. (1988). Statistical power analysis for the behavioral sciences (2 ed.). Hillsdale, NJ: Lawrence Earlbaum Associates.

Conselho Federal de Psicologia. (2007). Resolução CFP N. ${ }^{o}$ 013/2007 - Consolidação das resoluções relativas ao título profissional de especialista em Psicologia. Brasília: CFP.

Ferreira, A. I., Rodrigues, R. I., \& Ferreira, P. C. (2015). Career interests of students in psychology specialties degrees: psychometric evidence and correlations with the RIASEC dimensions. International Journal for Educational and Vocational Guidance, 16(1), 91-111. doi: 10.1007/ s10775-015-9289-3

Haselhuhn, C., \& Clopton, K. (2008). The representation of applied psychology areas in introductory psychology textbooks. Teaching in Psychology, 35(3), 205-209. doi: 10.1080/00986280802189130
Instituto Nacional de Estudos e Pesquisas Educacionais Anísio Teixeira. (2014). Censo da Educação Superior 2012: resumo técnico. Brasília: INEP.

Lamas, K. C. A., Ambiel, R. A. M., \& Silva, B. T. A. O. L. (2014). Vivências acadêmicas e empregabilidade de universitários em final de curso. Temas em Psicologia, 22(2), 329-340. doi: 10.9788/TP2014.2-05

Lemos, A. H. C., Neves, D. R., \& Rodrigues, P. R. F. (2013). Inserção de alunos bolsistas no mercado de trabalho: qual o valor do diploma universitário? Revista Pensamento Contemporâneo em Administração, 7(4), 24-41. Recuperado de http://search.proquest.com/ openview/a1f4bb85c5f665db8c051b165aa2a07b/1? pq-origsite $=$ gscholar\&cbl $=2032639$

Lent, RW., Brown, S.D., \& Hackett, G. (1994). Toward a unifying social cognitive theory of career and academic interest, choice, and performance. Journal of Vocational Behavior, 45, 79-122. doi: 10.1006/jvbe.1994.1027

Lopes, M. P., Palma, P. J., Bártolo-Ribeiro, R., \& Cunha, M. P. (2011). Psicologia aplicada. Lisboa, Portugal: Editora RH.

Magalhães, M. O. (2013). Sucesso e fracasso na integração do estudante à universidade: um estudo comparativo. Revista Brasileira de Orientação Profissional, 14(2), 215226. http://pepsic.bvsalud.org/pdf/rbop/v14n2/07.pdf

Magalhães, M. O., \& Teixeira, M. A. P. (2013). Antecedentes de comportamentos de busca de emprego na transição da universidade para o mercado de trabalho. Psicologia: Teoria e Pesquisa, 29(4), 411-419. http://www.scielo.br/ $\mathrm{pdf} / \mathrm{ptp} / \mathrm{v} 29 \mathrm{n} 4 / \mathrm{v} 29 \mathrm{n} 4 \mathrm{a} 07$

Magalhães, M. O., Straliotto, M., Keller, M., \& Gomes, W. B. (2001). Eu quero ajudar as pessoas: a escolha vocacional da psicologia. Psicologia: Ciência e Profissão, 21(2), 10-27. doi: 10.1590/S1414-98932001000200003

Martins, G. H., \& Ambiel R. A. M. (2015). Evidências de validade para a Escala de Interesses por Áreas da Psicologia. Trabalho apresentado na modalidade oral, no VII Congresso Brasileiro de Avaliação Psicológica, Universidade Presbiteriana Mackenzie, São Paulo, SP.

Martins, G. H., Hernández, D. N., \& Ambiel, R. A. M. (2014). Validade de conteúdo para a Escala de Interesses por Áreas da Psicologia. Trabalho apresentado na modalidade painel, no IV Congresso Brasileiro Psicologia: Ciência e Profissão, Uninove, São Paulo, SP.

Ministério da Educação. (2011). Diretrizes Curriculares Nacionais para o Ensino Médio. Brasília: MEC, Conselho Nacional de Educação/Câmara de Educação Básica.

Ministério da Educação. (2014). Relatório de Gestão do Exercício 2013. Brasília: MEC, Secretaria Executiva.

Neves, C. E. B. (2012). Ensino superior no Brasil: expansão, diversificação e inclusão. In LASA 2012/XXX International Congress of the Latin American Studies Association. São Francisco, EUA. 
Noronha, A. P. P., \& Rappold, C. T. (2010). Considerações sobre a avaliação psicológica no Brasil. Psicologia: Ciência e Profissão, 30(núm. esp.), 192-201. https:// www.researchgate.net/profile/Ana_Noronha3/ publication/262511324_Considerations_ about_Psychological_assessment_in_Brazil/ links/55098d140cf27e990e0ef151.pdf

Noronha, A. P. P., Ambiel. R. A. M., Frigatto, V., \& Toledo, C. C. R. (2010). Relações entre interesses, intenções e critérios de escolha profissional. Estudos Interdisciplinares em Psicologia, 1(1), 2-25. Recuperado de http://pepsic. bvsalud.org/pdf/eip/v1n1/a02.pdf

Rodrigues, R. I., Ferreira, A. I., \& Bártolo-Ribeiro, R. (2013). Construção e desenvolvimento de um questionário de interesses para a psicologia. Iberoamericana de Diagnóstico y Evaluación/e Avaliaçao Psicológica, 36,99-116.

Rounds, J., \& Su, R. (2014). The nature and power of interests. Current Directions in Psychological Science, 23(9), 98-103. doi: 10.1177/0963721414522812

Sarriera, J. C., Paradiso, A. C., Schütz, F. F., \& Howes, G. P (2012). Estudo comparativo da integração ao contexto universitário entre estudantes de diferentes instituições. Revista Brasileira de Orientação Profissional, 13(2),163-
172. Recuperado de http://pepsic.bvsalud.org/pdf/rbop/ v13n2/04.pdf

Silva, C. S. C., Coelho, P. B. M., \& Teixeira, M. A. P. (2013). Relações entre experiências de estágio e indicadores de desenvolvimento de carreira em universitários. Revista Brasileira de Orientação Profissional, 14(1), 3546. Recuperado de http://pepsic.bvsalud.org/pdf/rbop/ v14n1/05.pdf

Silvia, P. J. (2001). Expressed and measured vocational interests: distictions and definitions. Journal of Vocational Behavior, 59(3), 382-393. doi: 10.1006/jvbe.2001.1805

Super, D. E., Savickas, M. L., \& Super, C. M. (1996). The life-span, life-space approach to careers. In D. Brown, L. Brooks \& Associates (Eds.), Career choice \& development, (3 ed.,pp.121-178). São Francisco, EUA: Jossey-Bass.

Teixeira, M. A. P., \& Gomes, W. B. (2004). Estou me formando... e agora? Reflexões e perspectivas de jovens formandos universitários. Revista Brasileira de Orientação Profissional, 5(1), 47-62. Recuperado de http://pepsic. bvsalud.org/pdf/rbop/v5n1/v5n1a05.pdf

Yamamoto, O. H., \& Costa, A. L. F. (2010). Escritos sobre a profissão de psicólogo no Brasil. Natal, RN: EDUFRN. 